\title{
MANTLE METASOMATISM IN MARS: POTASSIC BASALTIC SANDSTONE IN GALE CRATER DERIVED FROM PARTIAL MELT OF PHLOGOPITE PERIDOTITE
}

Allan H. Treiman, Lunar \& Planetary Institute Etienne Medard, Blaise Pascal Univ. \& LPI 


\section{Introduction}

- Rocks of Gale crater are alkali-rich, compared to martian meteorites* \& most other in situ analyses.

- Basaltic sandstones of Kimberley area are especially rich in potassium (to $6 \% \mathrm{~K}_{2} \mathrm{O}$ ); suggested (Treiman et al. 2016) a trachytic sediment source.

- Alternate idea here, that the K-rich sediment represents a potassic basalt, such as would form by partial melting of from a phlogopite-bearing (metasomatized) mantle peridotite.

* Except a few K-rich melt inclusions in nakhlites (Goodrich et al. 2013) 


\section{Kimberley Area Geology}
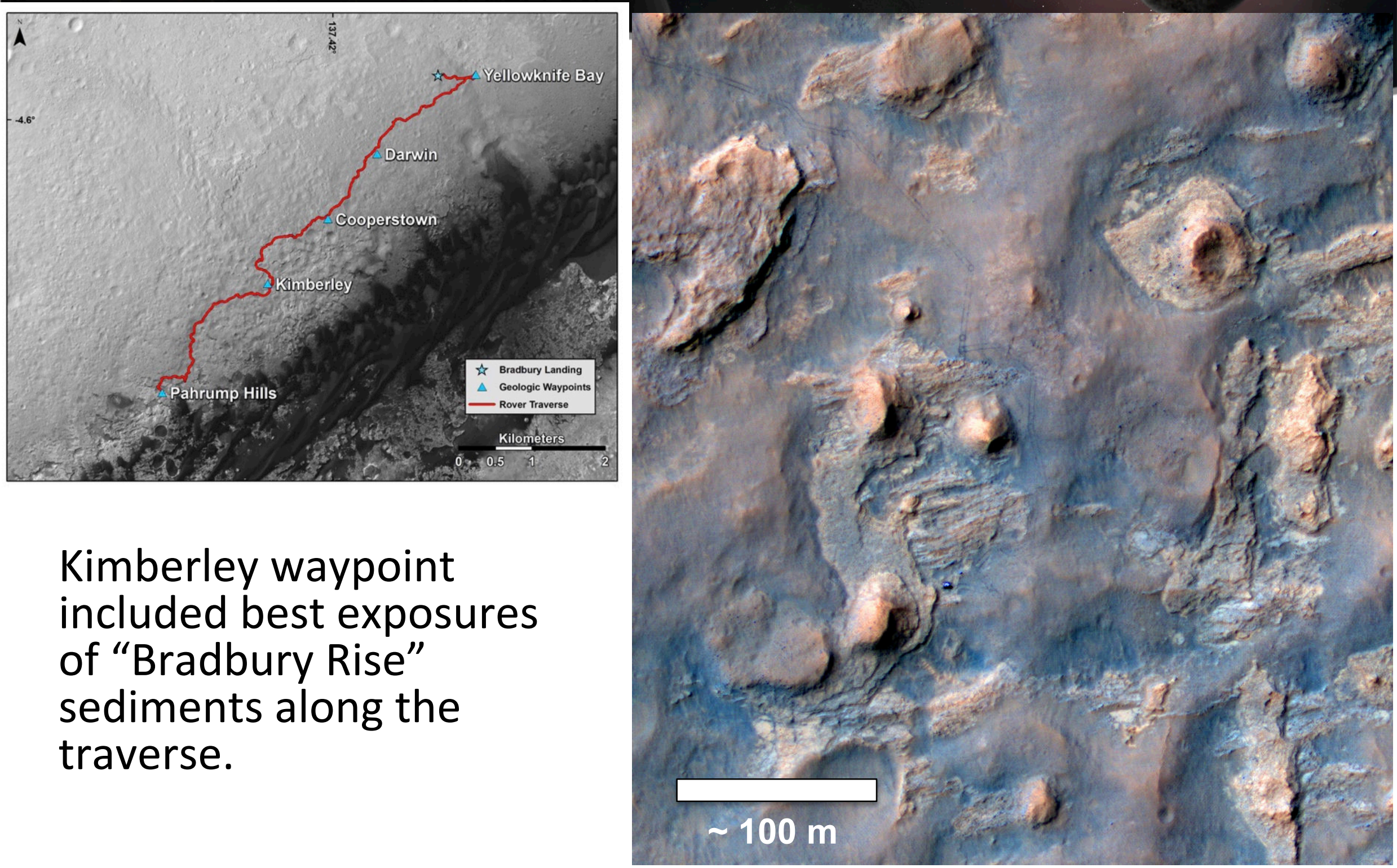

Kimberley waypoint included best exposures of "Bradbury Rise" sediments along the traverse. 


\section{Kimberley Area Outcrops}

Mt.

Remarkable

A fluvio-deltaic sequence, shed off Gale Crater walls. Unit with undulating beds (Square Top) is as deltaic clinoforms. Overlying unit (Dillinger) represents high flow regime, i.e. antidune structures (Gupta, 2016, this meeting).

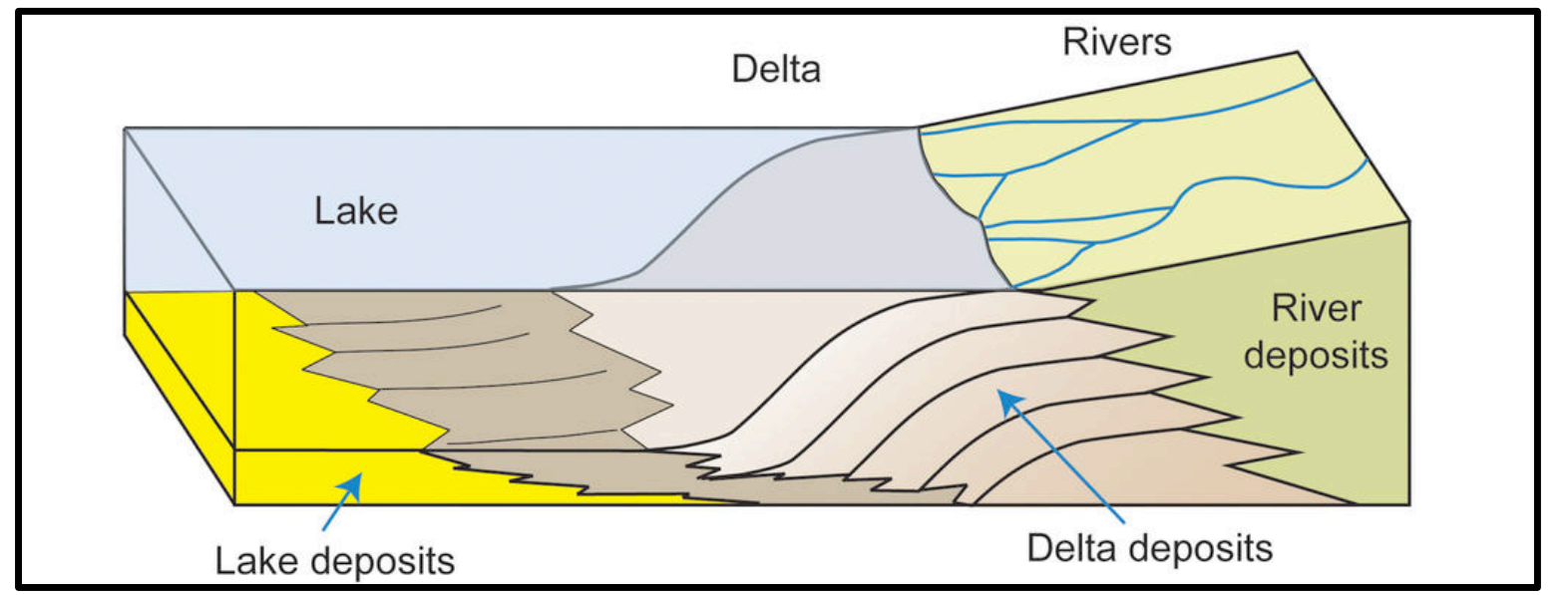




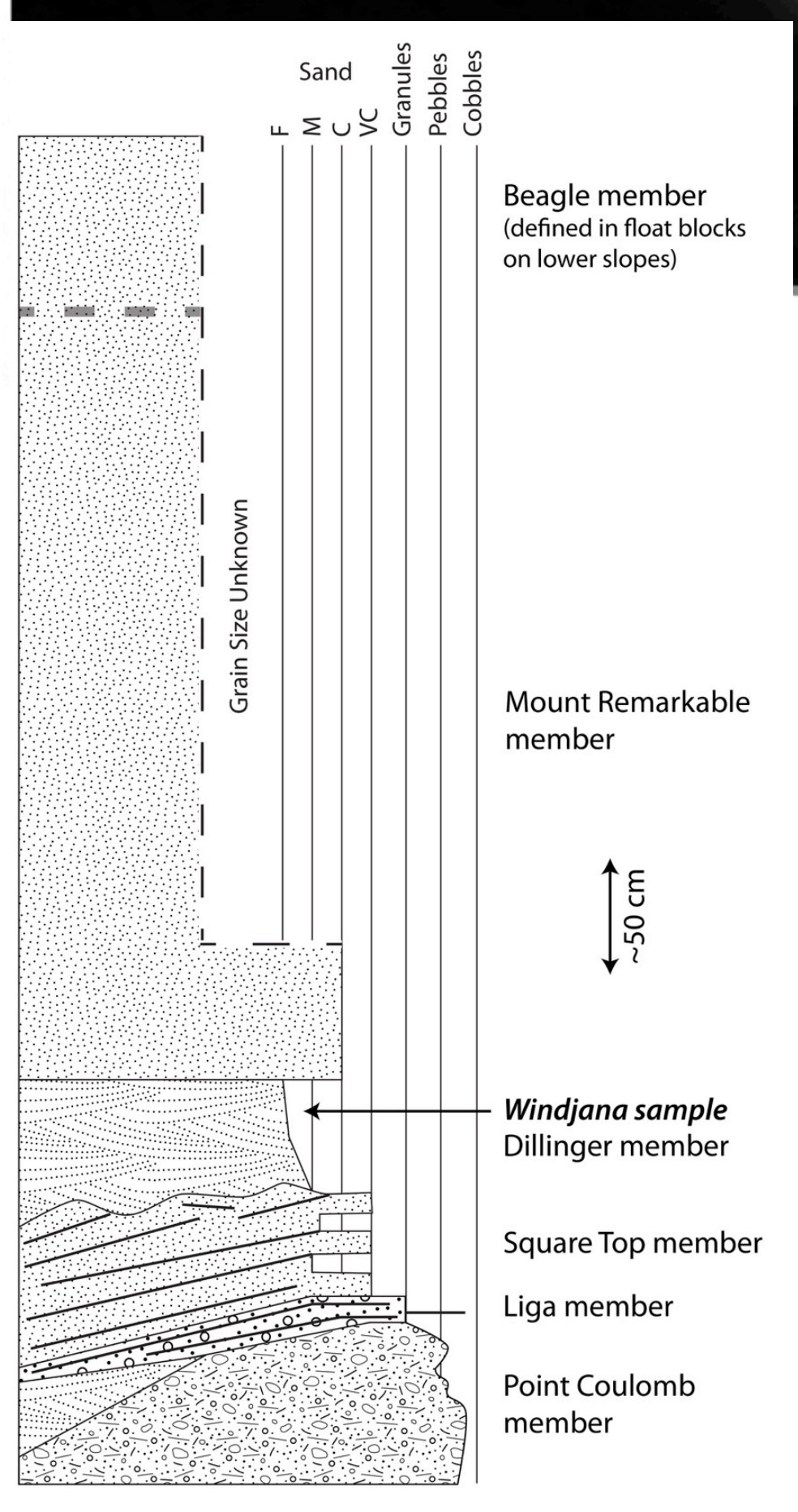

\section{Kimberley Area Stratigraphy}

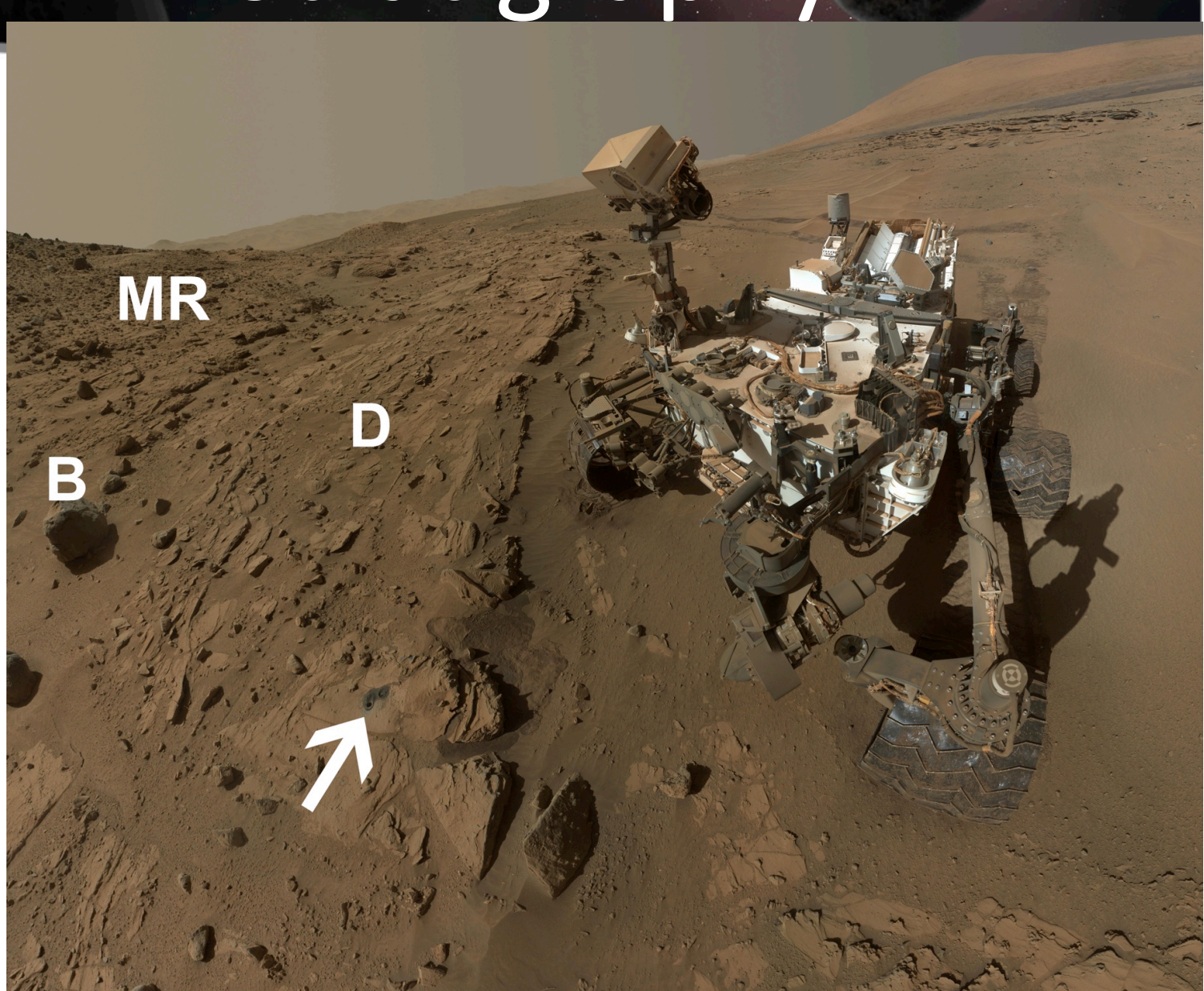

MAHLI Selfie at Windjana dril site (arrow). Square_Top oucrops behind Curiosity. Dillinger and Mt. Remarkable members to left. Fallen boulder of Beagle (capping unit) at far left. 


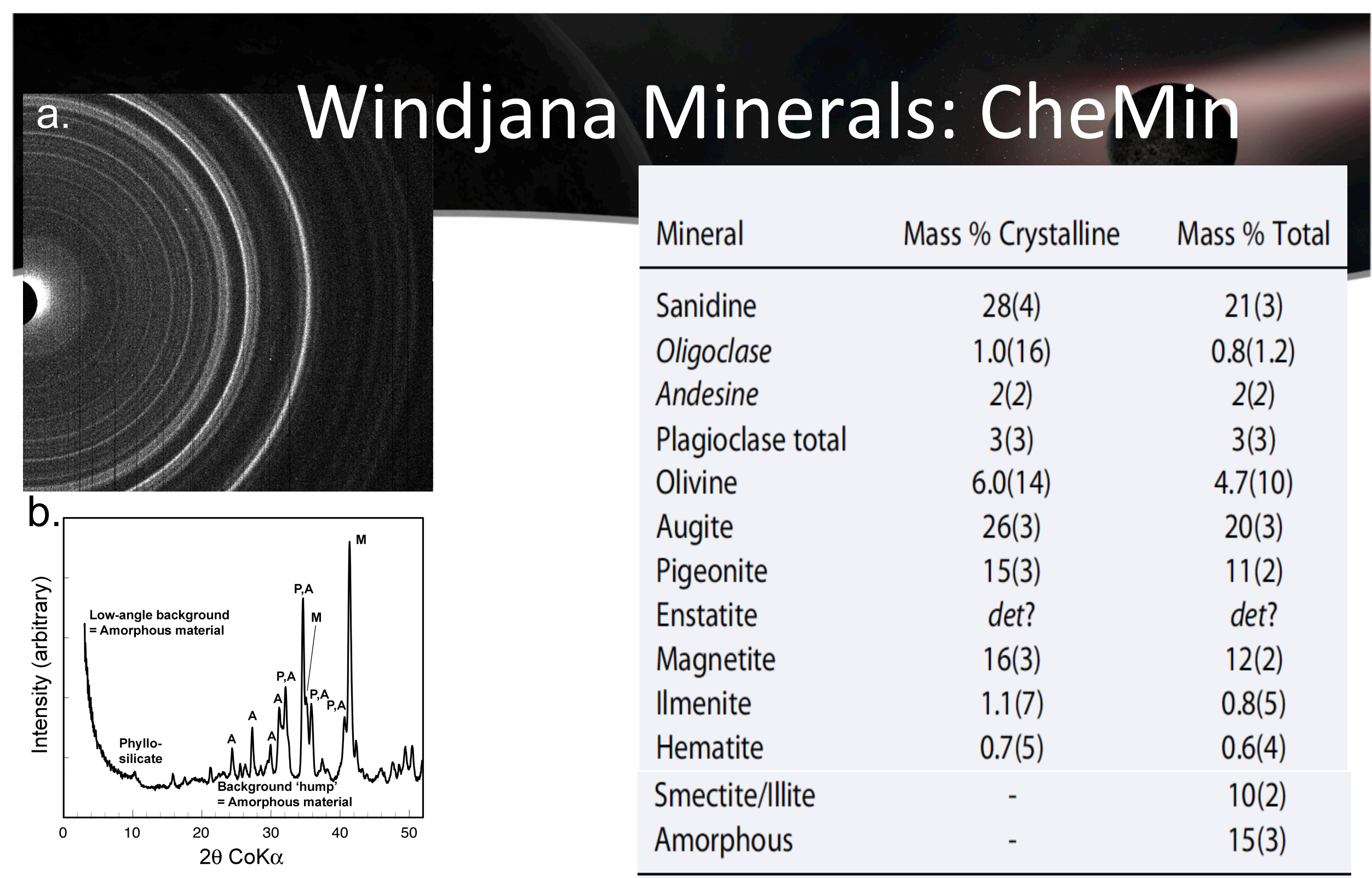

Sanidine is near-endmember, disordered $\mathrm{KAISi}_{3} \mathrm{O}_{8}$. Small proportions of secondary/aqueous minerals. (Treiman et al. 2016). 


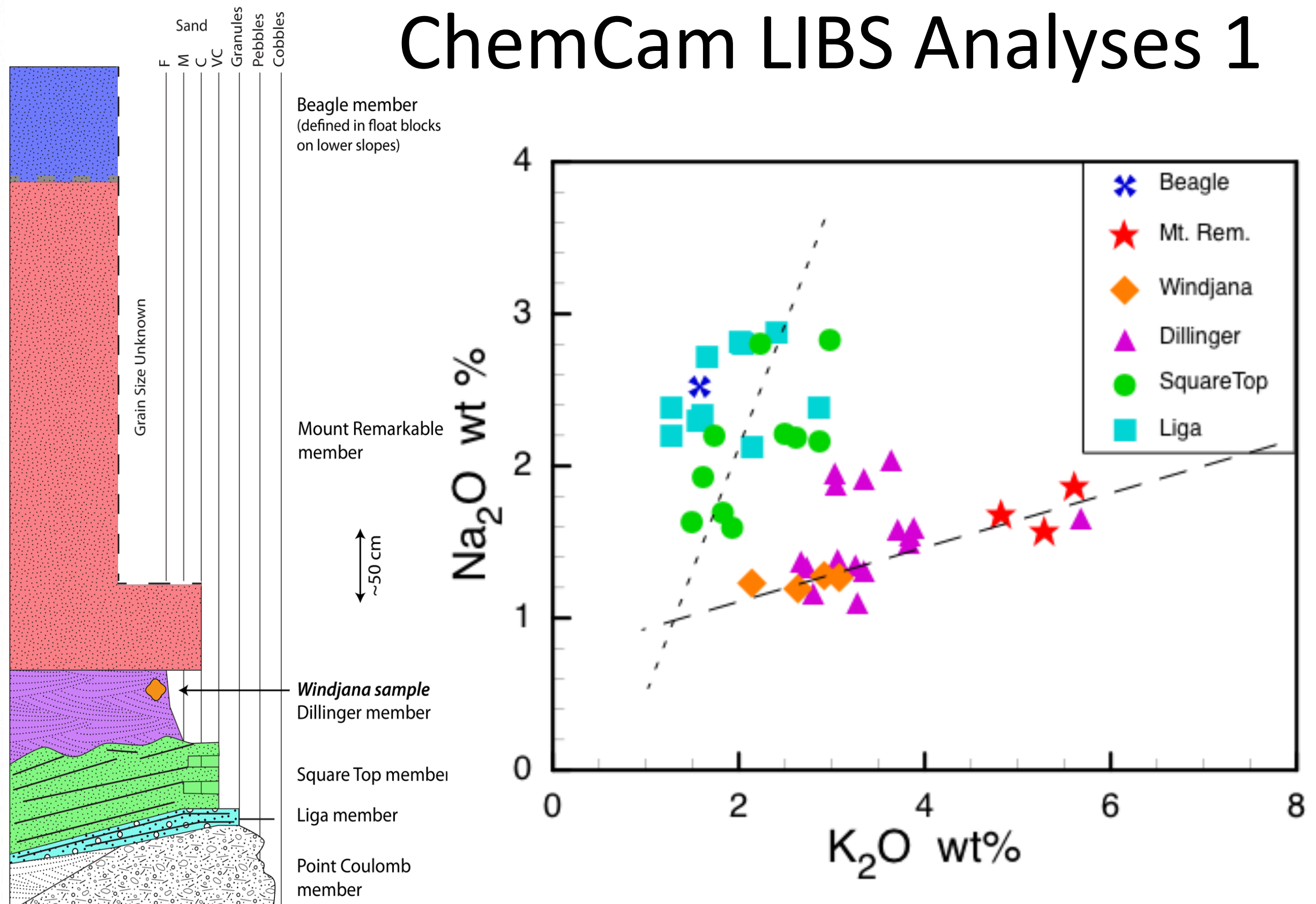




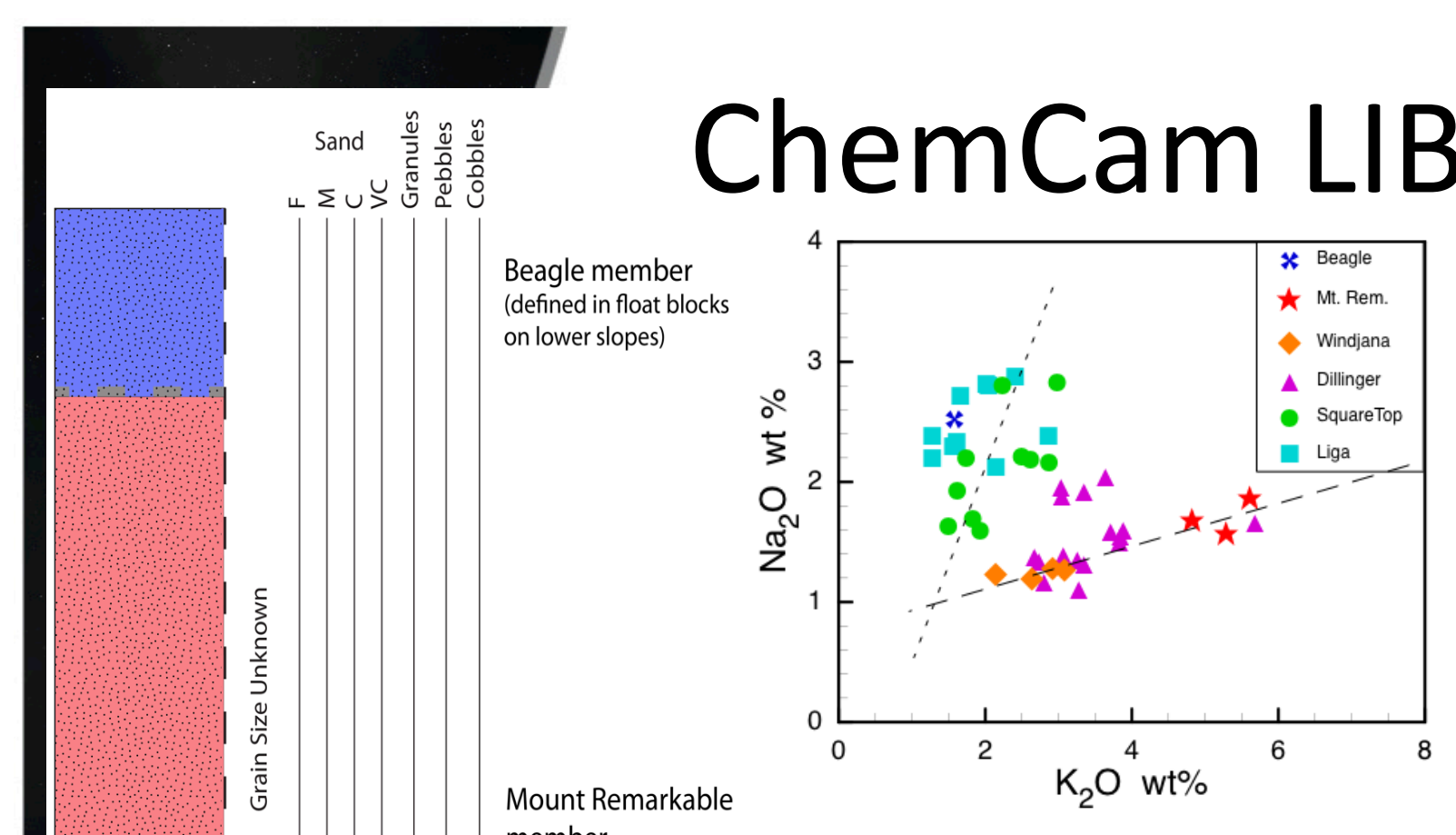

\section{ChemCam LIBS Analyses 2}

member
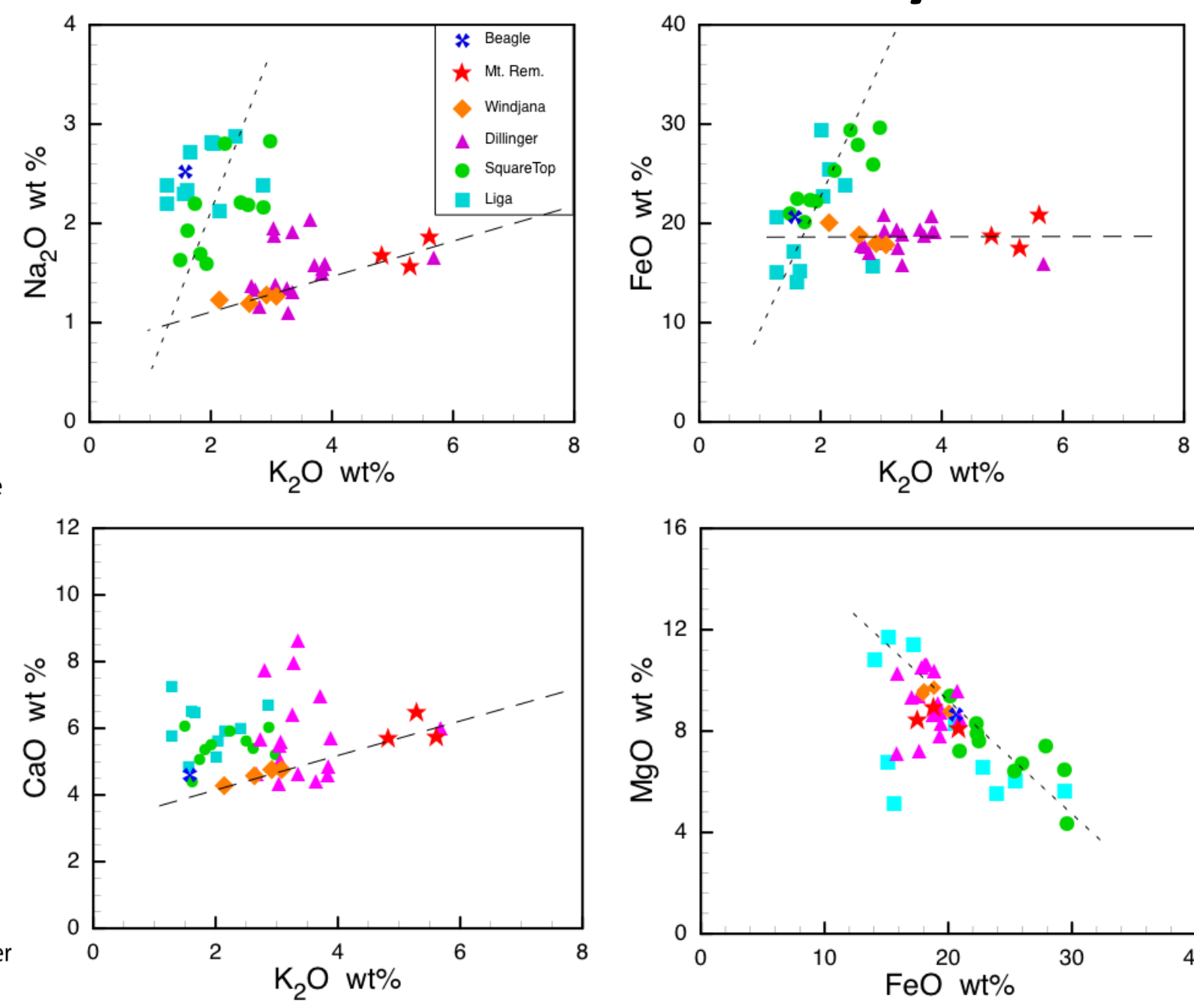

Windjana sample Dillinger member

Square Top member

Liga member

Trend-lines for Dillinger-Mt._Remarkable Point Coulomb member compositions do not extend towards trachyte. What does Mt._Remarkable represent?

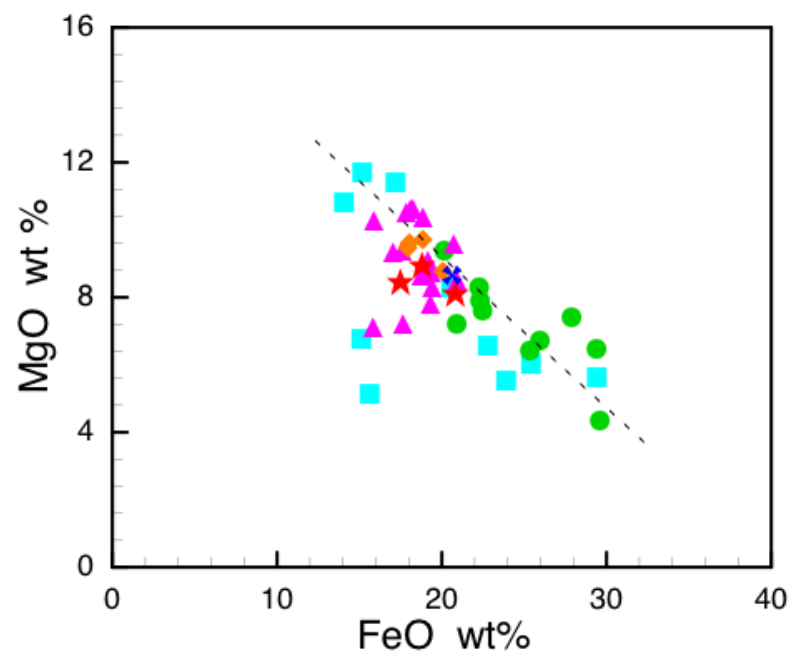




\section{Leaps of Faith (ein Ausflug ins Blaue)}

- Sandstone compositions represent mixtures of igneous rock types.

- Little evidence of non-isochemical alteration.

- No evidence to suggest mineral fractionation during sediment transport or deposition.

- ChemCam LIBS analyses are accurate.

- I.e., they are directly comparable with XRF and EMP analyses of Earth and laboratory materials. 


\section{Mt._Remarkable Composition}

- Like other martian basalts, but leucite-normative!

\begin{tabular}{|c|c|c|c|c|}
\hline & Gale & \multicolumn{3}{|c|}{ Gusev } \\
\hline & "Mt_Rem" & Fastball & Backstay & Adirondak \\
\hline & CCAM & APXS-MER & APXS-MER & APXS-MER \\
\hline $\mathrm{SiO}_{2}$ & 48.0 & 48.7 & 49.5 & 45.9 \\
\hline $\mathrm{TiO}_{2}$ & 1.0 & 0.7 & 0.9 & 0.6 \\
\hline $\mathrm{Al}_{2} \mathrm{O}_{3}$ & 11.0 & 8.4 & 13.3 & 10.6 \\
\hline $\mathrm{FeO}_{\mathrm{T}}$ & 17.0 & 19.1 & 13 & 18.7 \\
\hline $\mathrm{MgO}$ & 9.0 & 12.9 & 8.3 & 9.9 \\
\hline $\mathrm{CaO}$ & 6.3 & 6.2 & 6 & 7.9 \\
\hline $\mathrm{Na}_{2} \mathrm{O}$ & 1.7 & 2.5 & 4.2 & 2.6 \\
\hline $\mathrm{K}_{2} \mathrm{O}$ & $\equiv 6.0$ & 0.3 & 1.1 & 0.2 \\
\hline
\end{tabular}




\section{Mt._Remarkable Composition - TAS}

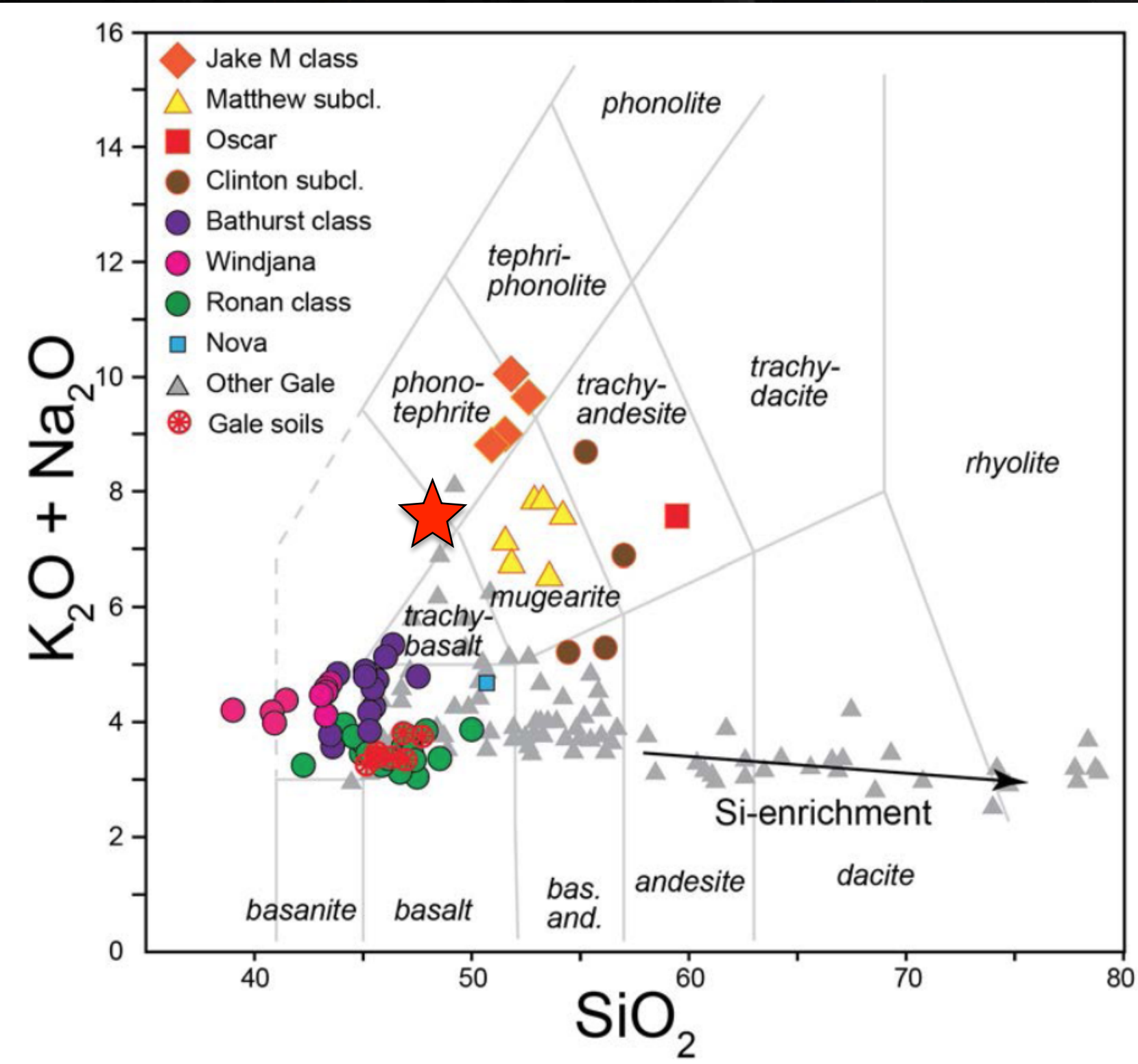

From M. Schmidt, previous talk 


\section{Peridotite Partial Melt}

- Bulk is comparable to partial melts of martian mantle peridotite, Dreibus \& Wänke 1984 (Collinet et al. 2015).

- Potassium content consistent with partial melts of phlogopitebearing peridotite (Condamine \& Medard 2014).

\begin{tabular}{|c|c|c|c|c|}
\hline & $\begin{array}{c}\text { Gale } \\
\text { “Mt_Rem” }\end{array}$ & $\begin{array}{l}\text { Collinet } \\
\text { DW-84 }\end{array}$ & $\begin{array}{l}\text { Collinet } \\
\text { DW-84 }\end{array}$ & $\begin{array}{c}\text { Condamine } \\
\text { Phl-Hzb }\end{array}$ \\
\hline & CCAM & $1.5 \mathrm{GPa} 1300^{\circ} \mathrm{C}$ & $2 \mathrm{GPa} 1375^{\circ} \mathrm{C}$ & $1 \mathrm{GPa} 1200^{\circ} \mathrm{C}$ \\
\hline $\mathrm{SiO}_{2}$ & 48.0 & 47.4 & 46.4 & 56.0 \\
\hline $\mathrm{TiO}_{2}$ & 1.0 & 0.8 & 0.8 & 1.5 \\
\hline $\mathrm{Al}_{2} \mathrm{O}_{3}$ & 11.0 & 12.8 & 10.8 & 18.4 \\
\hline $\mathrm{FeO}_{\mathrm{T}}$ & 17.0 & 14.7 & 16.6 & 4.7 \\
\hline $\mathrm{MgO}$ & 9.0 & 9.1 & 10.8 & 7.0 \\
\hline $\mathrm{CaO}$ & 6.3 & 8.7 & 8.7 & 5.1 \\
\hline $\mathrm{Na}_{2} \mathrm{O}$ & 1.7 & 4.2 & 3.6 & 1.0 \\
\hline $\mathrm{K}_{2} \mathrm{O}$ & $\equiv 6.0$ & 0.5 & 0.5 & 6.4 \\
\hline
\end{tabular}




\section{Conclusions}

- Sandstone of Kimberley area were derived from several basaltic sources.

- The most potassic sediment source, $\mathrm{K}_{2} \mathrm{O} \geq 6 \%$, was likely a Lc-normative basalt, not a trachyte.

- The inferred composition of the potassic sediment source is consistent, in general, with a primary melt from a phlogopite-bearing (metasomatized) mantle. 